\section{Protein phosphorylation and LHCII structure}

A recent article in TIBS by John F. Allen on the role of protein phosphorylation in the regulation of photosynthesis attracted our attention ${ }^{1}$. In his article, the author seemed to suggest that this role could be explained by the molecular recognition hypothesis. We would like to comment on some aspects of this hypothesis.

The author suggests that phosphorylation of Thr6 in light-harvesting complex II (LHCII) induces helicity in the amino-terminal segment by increasing the net negative charge and consequently screening the repulsion between the positively charged residues in the region. To test this proposal, we replaced Thr6 with Glu and subjected the first 20 amino acids of the amino-terminal segment of LHCII to secondary structure prediction using Garnier's method. As a consequence, the helical content increased from $35 \%$ to $45 \%$. This increase may not be significant when the uncertainty in the predicted values is considered. Studies by Padmanabhan et al. ${ }^{2}$ suggest that the propensity of a sequence to form a helix depends on the sequence context. Therefore, we feel that the amino-terminal segment could have an inherent ability to adopt a helical conformation and phosphorylation only imparts additional stability to the helix formed.

Allen goes on to suggest that when Thr6 is in its unphosphorylated form, the positively charged residues located within 10-12 A repel each other and introduction of a phosphate group diminishes this repulsion. We would like to mention that repulsion between positive charges situated $10-12 \AA$ apart in a helix would only be marginal. In fact, we generated an $\alpha$-helix with the first 13 amino-terminal residues of LHCII and calculated the charge-charge interaction energy between side chains of different positively charged amino acids using AMBER force-field parameters. We found that electrostatic repulsion is negligible when charged groups are more than $10 \AA$ apart in the helix, but is very significant when they are situated within 5-6.̊. Thus, we feel that if phosphorylation induces helicity, its effects would be felt more by way of stabilization of the helix through salt bridges between phosphate groups and side chains of positively charged amino acids on both sides, rather than by screening the repulsion Sundaralingham et $a l^{3}$ showed the formation of salt bridges between oppositely charged residues located at $i$ and $i \pm 3$ or $i \pm 4$ positions imparts additional stability to the helix. Such a possibility exists in LHCII

(MRKSATTKKVASS, where $\mathbf{T}=\mathbf{i}$ ).

We understand that the repulsion screening hypothesis derives its strength basically from the structural changes reported in glycogen phosphorylase after appropriate to stretch the analogy too far. To the best of our knowledge, there is no report so far to suggest that phosphorylation of an amino acid in model synthetic peptides leads to drastic changes in secondary structure. We hope that structural studies on the phosphorylated and dephosphorylated amino-terminal segment of LHCII will throw light on the validity of the proposal. between them. In this context, phosphorylation, and believe that it is not

We would like to point out that the hypothesis does not take into account the possible role of phosphorylation in structural changes of other photosynthetic membrane proteins and PSII. We also feel that multiple phosphorylation is another aspect that deserves a place in the hypothesis.

\author{
References \\ 1 Allen, J. F. (1992) Trends Biochem. Sci. 17 , \\ 12-17 \\ 2 Padmanabhan, S. et al. (1990) Nature 344 \\ 268-270 \\ 3 Sundaralingam, M., Sekharudu, Y. C., \\ Yathindra, N. and Ravichandran, V. (1987) \\ Proteins 2, 64-71
}

\section{K. GOPAL KRISHNA, T. K. SURESH KUMAR AND M. W. PANDIT}

Centre for Cellular and Molecular Biology, Hyderabad 500 007, India.

\section{Reply from Allen}

As described in my Open Question article $^{1}$, the molecular recognition hypothesis makes a number of testable predictions. I too have run computer structural predictions to see if replacement of Thr6 with Glu might mimic the proposed effect of phosphorylation ${ }^{2}$. I find that helix probability increases for each amino acid from 1 to 11 . This is quite consistent with a small increase in a moderate helical content $(35 \%)$ for the first 20 amino acids of LHCIlb. A control 'experiment' is to apply the same predictions to the amino-terminal segment of glycogen phosphorylase with the corresponding substitution of Glu for Ser14. This produces a similar result ${ }^{2}$, and for glycogen phosphorylase helix formation is, of course, the correct answer ${ }^{3}$. It should also be noted that one might expect only moderate helix probability in any segment where the helix must be broken and remade. There is also the question of how well glutamate mimics effects of phosphothreonine and phosphoserine, in both computer programs and the real world.

I agree that a suitable initial approach is with structural studies on model peptides. Landry et al. report differences in nuclear Overhauser effects in two-dimensional nuclear magnetic resonance (NMR) spectra of the vsv-C peptide bound to DnaK and GroEL ${ }^{4}$. The spectra indicate that vsv-C is helical when bound to GroEL but in an extended conformation when bound to DnaK. The vsv-C peptide contains the sequence VLSSL, reminiscent of the sequence VASSG, which starts four positions after the LHCII phosphorylation site. It is possible to imagine that this is an 'optional helix' segment.

Krishna, Kumar and Pandit appear to favour helix formation by suggesting, later in their letter, that a helix may be stabilized by salt bridges between the phosphate and basic side chains at $\mathrm{i}+4$, $i-3$ and $i+3$. I do not exclude this possibility, though there is no precedent for it in glycogen phosphorylase ${ }^{3}$. In addition, it appears that engineered salt bridges between $i$ and $i+4$ have only marginal effects on stabilization of $\alpha$-helices ${ }^{5}$. In my article ${ }^{1}$ I propose salt bridges with more distant side chains as the basis of a tertiary structural change, though effects on secondary structure cannot be excluded. For LHCII these are all open questions.

I am pleased to acknowledge the questions raised in the letter of Krishna et al. I agree that phosphorylation of other thylakoid polypeptides must be taken into account when considering $\mathrm{LHCII}^{2}$. My chief objective in writing the Open Question article ${ }^{1}$ was to draw attention to the possibility of an LHCII 
protein structural change as an alternative to effects on thylakoid surface charge, and in so doing, to see how far one might usefully push an analogy with glycogen phosphorylase. I am delighted to think that questions of protein phosphorylation in general are beginning to focus on the forces stabilizing different states of protein folding. For thylakoid proteins this is an entirely new way of looking at the problem.

\section{References}

1 Allen, J. F. (1992) Trends Biochem. Sci. 17, 12-17

2 Allen, J. F. (1992) Biochem. Biophys. Acta 1098, 275-335

3.Barford, D., Hu, S-H. and Johnson, L. N. (1991)
J. Mol. Biol. $218,233-260$

4 Landry, S. J., Jordan, R., McMacken, R. and Gierasch, L. M. (1992) Nature 255, 455-457 5 Yang, A-S. and Honig, B. (1992) Curr. Op.

Struct. Biol. 2, 40-45

\section{JOHN F. ALLEN}

Plant Cell Biology, Lund University, Box 7007 , S-220 07 Lund, Sweden.

\section{Nuclear targeting sequences}

In a recent article in TIBS, Dingwall and Laskey ${ }^{1}$ reported a consensus amino acid sequence which may provide a signature for the targeting of proteins to the nucleus. This motif consists of a cluster of two adjacent basic amino acids separated by any ten amino acids from a second cluster, in which three of the next five amino acids are also basic. These authors reported that this consensus is shared by a large number of known nuclear genes, including several yeast $R A D$ genes ( $R A D 1$, $R A D 2$ and $R A D 7$ ) known to be involved in nucleotide excision repair (NER) of $D_{N A}{ }^{2}$ and the $R A D 18$ gene, which is required for DNA damage-induced mutagenesis ${ }^{2}$.

Many other yeast genes involved in the repair of damaged DNA have been cloned and sequenced. The amino acid sequences of several human excision repair genes have also been reported $^{3}$. I have identified consensus nuclear targeting sequences (in some cases more than one in a given polypeptide) in the yeast NER genes RAD4 and RAD14 and in the human NER gene ERCC3 (Table I). The consensus sequence in the ERCC3 gene is conserved in the homologous yeast SSL2 gene ${ }^{4}$. The yeast SNMI (PSO2) gene, which is specifically required for the excision repair of crosslinks in DNA ${ }^{2}$, also contains a consensus motif. This sequence is additionally present in the yeast $R A D H, R E V 1$ and $R E V 3$ genes, which like $R A D 18$, are required for damageinduced mutagenesis ${ }^{2}$ and the $P H R 1$ genes, which is required for the enzymatic photoreactivation of pyrimidine dimers in $\mathrm{DNA}^{2}$. While it is evident that the consensus sequence identified by Dingwall and Laskey ${ }^{1}$ is conserved in more eukaryotic DNA repair proteins than originally identified, it is to be noted that a number of other polypeptides that are known to be involved in cellular responses to DNA damage in yeast and human cells do not contain this domain.

\section{References}

1 Dingwall, C. and Laskey, R. A. (1991) Trends Biochem. Sci. 16, 478-481
Table I. Bipartite nuclear transport sequences in eukaryotic DNA repair genes

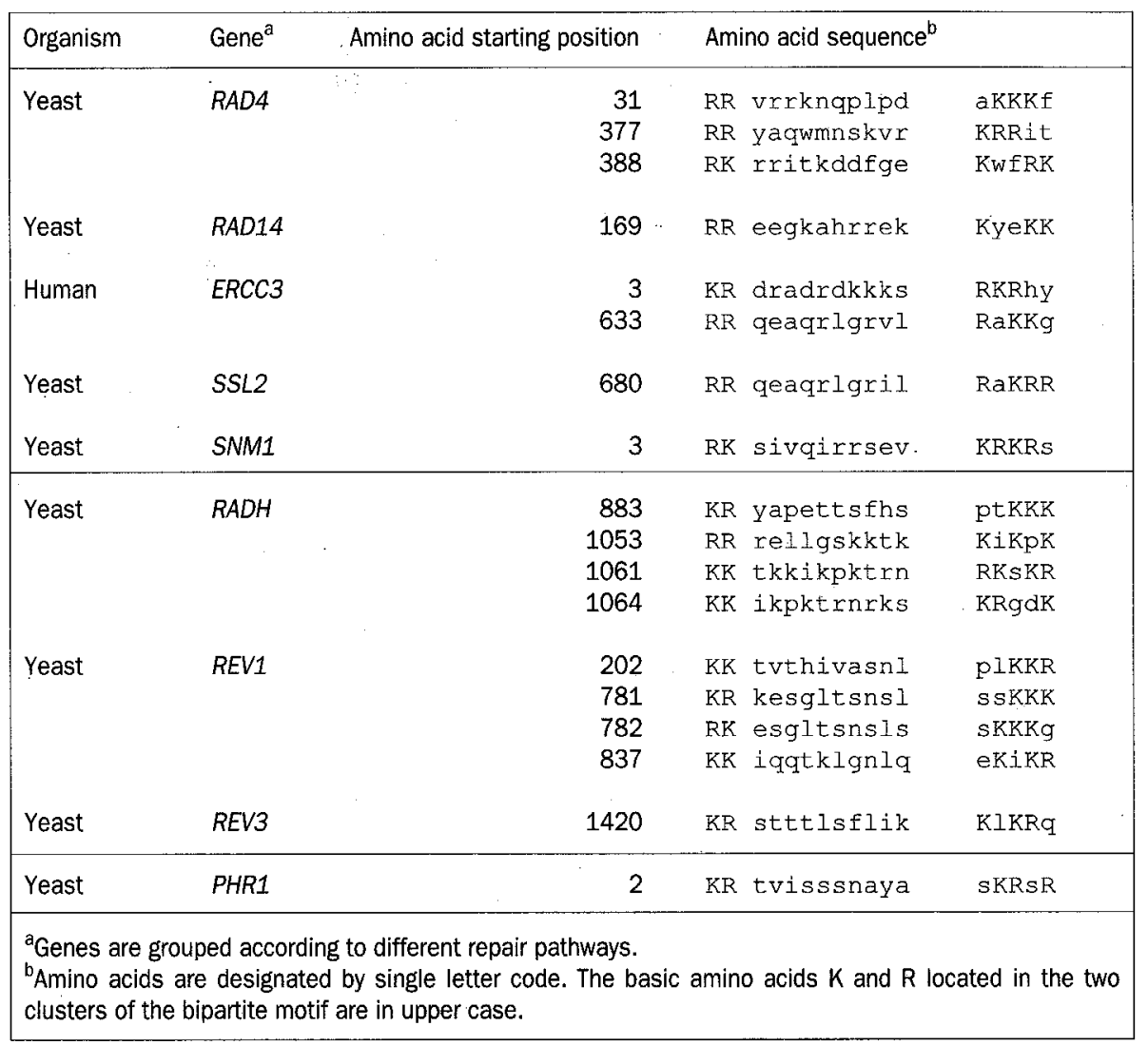

2 Friedberg, E. C. et al. in The Molecular and Cellular Biology of the Yeast Saccharomyces: Genome Dynamics, Protein Synthesis and Energetics (Broach, J., Jones, E. and Pringle, J., eds), pp. 147-192, Cold Spring Harbor Laboratory Press

3 Hoeijmakers, J. H. J. and Bootsma, D. (1990) Cancer Cells 2, 311-320
4 Gulyas, K. D. and Donahue, T. F. (1992) Cell $69,1031-1042$

\section{ERROL C. FRIEDBERG}

Laboratory of Molecular Pathology, Department of Pathology, University of Texas Southwestern Medical Center at Dallas, Dallas, TX 75235, USA.

\section{Letters to TIBS}

TIBS welcomes letters on any topic of interest. Please note, however, that previously unpublished data and criticisms of work published elsewhere cannot be accepted by this journal.

Letters should be sent to:

\author{
Trends in Biochemical Sciences \\ Elsevier Trends Journals, \\ 68 Hills Road, \\ Cambridge, UK CB2 1LA.
}

